\title{
A STUDY ON CLINICAL EFFECT OF COMBINED PULSED DYE LASER AND INTRALESIONAL TRIAMCINOLONE ACETONIDE IN THE TREATMENT OF KELOID
}

\author{
YAZDI QS ${ }^{1}, \mathrm{KHAN} \mathrm{AL}^{2}, \mathrm{HASAN} \mathrm{MS}^{3}, \mathrm{MURAD} \mathrm{IH}^{4}$
}

\begin{abstract}
:
Keloids are common and cause functional and psychological morbidity. A wide variety of treatments, all in current usage, indicate that no treatment has been shown to be markedly superior to the others. This study was done using pulsed dye laser followed by more traditional intralesional steroid injection in the treatment of keloid. The aim of this study was to determine the effectiveness of the PDL in combination with intralesional triamcinolone acetonide (TCA) in the treatment of keloid and to elucidate possible side effects and complications. A prospective clinical trial was done in the Department of Dermatology \& Venereology, Combined Military Hospital, Dhaka Cantonment, Dhaka, from December 2013 to February 2014. Adult patients of both sexes (Fitzpatric skin type IV \& V) aged between 15 and 50 years with varying degree of keloids were randomly selected for the study. In this single-blinded clinical trial, 50 patients were randomly assigned and was irradiated by 595- $\mathrm{nm}$ flashlamp-pumped pulsed-dye laser $(P D L, 5-7.5 \mathrm{~J} / \mathrm{cm} 2)$ at the $1^{\text {st }}, 4 \mathrm{th}$, and 8 th weeks and Intralesional steroid was given following PDL. Lesions were assessed for vascularity, pliability, pigmentation and height. The study showed an excellent improvement in nearly all measures, Good to excellent improvements (>76\% improvement) were reported by $93 \%$ patient. Vancouver scar scale (0-14) decreased from 12.17 to a post treatment value of 3.41 which is an excellent achievement. Results were reported by the blinded observer as follows: in vascularity (from 2.7 to 0.61), in pliability (from 3.9 to 1.1), in hyperpigmentation (from 2.4 to 0.92), in height (from 2.87 to 0.78).
\end{abstract}

Key words: Keloid, pulsed dye laser, intralesional triamcinolone acetonide.

J Dhaka Med Coll. 2014; 23(2) : 234-238.

\section{Introduction:}

Cutaneous dermal injury eventuates in the inevitable formation of a keloid, which may be cosmetically unacceptable. ${ }^{1}$ Keloids are dermal fibroproliferative disorders unique to human that occur following trauma, inflammation, surgery, burn and sometimes spontaneously. These are characterized by excess deposition of collagen in the dermis and the subcutaneous tissues, the exuberant scarring of keloid results typically in disfigurement, contractures, pruritis and pain. Keloids occur in individuals with a familial disposition among the Blacks, Hispanics and Orientals, they enlarge and extend beyond the margins of the original wound and rarely regress. These disorders represent aberrations in the fundamental processes of wound healing, which include cell migration and proliferation, inflammation, increased synthesis and secretion of cytokines and extra cellular matrix $(\mathrm{ECM})$ proteins and remodeling of the newly synthesized matrix ${ }^{2,6}$

Despite increasing knowledge of wound healing and collagen metabolism over the past decade, keloids traditionally been difficult lesions to eradicate. Prior to the discovery, that pulsed dye lasers could be used safely and effectively to treat keloid, treatments ranging from invasive surgical excisions and grafting procedures to dermabrasion, cryotherapy, corticosteroid injections, were used with varying degrees of success. Unfortunately,

1. Lt. Col. Quazi SalimYazdi, Combined Military Hospital, Dhaka Cantonment, Dhaka.

2. Col. Abdul Latif Khan, Combined Military Hospital, Dhaka Cantonment, Dhaka.

3. Col. Md SayeedHasan, Combined Military Hospital, Dhaka Cantonment, Dhaka.

4. Lt. Col. Imranul Hasan Murad, Combined Military Hospital, Dhaka Cantonment, Dhaka.

Correspondence: Lt Col Quazi SalimYazdi, Combined Military Hospital, Dhaka Cantonment, Dhaka. Email: yazdi886@hotmail.com. 
most of these treatments often were of little benefit or had side effects that were nearly as severe as the original keloid. . The more recent introduction of topical treatments, including retinoic acidand silicone gel, has produced waves of enthusiasm, but overall effect has been minimal and slow to achieve. ${ }^{3}$

Beginning in the late 1980s, experiments using a vascular-specific pulsed dye laser on hypertrophic scars present within port-wine stains were initiated. The laser-treated scar became more pliable and less hypertrophic, erythematous, and pruritic. This clinical observations were later substantiated by skin surface textural analyses, erythema reflectance spectrometryreadings, scar height measurements, and pliability scores, all showing significant improvement with one or two laser sessions. ${ }^{3,4} \mathrm{Histopathologic}$ examination of laser-irradiated keloids confirmed the suspected improvement in dermal collagen (more fine and fibrillar postlaser treatment), but also pointed to a possible etiologic explanation for the laser's effectiveness with the appearance of increased numbers of regional mast cells in the irradiated keloid, mast cells elaborate an intriguing variety of cytokines. Their presence following laser irradiation and accompanying tissue revascularization may provide an explanation for the therapeutic outcome. Other etiologic mechanisms include the possibility of collagen stimulation by dermal heat conduction from the irradiated blood vessels or lack of tissue oxygenation leading to collagen catabolism and release of collagenase. ${ }^{5,3}$ The high vascular specificity of the pulsed dye laser is no doubt responsible for the decreased keloid erythema, but the nonvascular improvements may best be explained by laser induced tissue hypoxia stimulating new collagen formation, and with the release or stimulation of various immunofactors enhance collagen remodeling. Prolonged follow-up of patients 6 months to several years after pulsed dye laser treatment has revealedno keloid worsening nor recurrences, further indicating the importance of this therapeutic modality. ${ }^{3}$

During each laser session, the entire keloid is treated with adjacent, nonoverlapping laser pulses at appropriate energy densities (or fluences). The spot size used, the color and thickness of the keloid and its location are the factors to determine the choice of energy density. During the early sessions, lower fluences are generally used. Depending upon the keloid response, the fluences are adjusted upward during subsequent treatments. Slightly higher fluences may be used to treat thicker or darker keloids while lower energy densities are used to treat pale, less fibrotic keloids in sensitive or thin-skinned areas (e.g. anterior chest, neck). ${ }^{1,4}$

\section{Methods:}

This is a prospective clinical trial, done in the Department of Dermatology \& Venereology, Combined Military Hospital, Dhaka Cantonment, Dhaka, from December 2013 to February 2014. Adult patients of both sexes (Fitzpatric skin type IV \& V) aged between 15 and 50 with varying degree of keloids were randomly selected for the study. A total of 50 patient who fulfilled the inclusion and exclusion criteria were included and were treated with pulsed dye laser followed by intralesional triamcinolone acetonide injection on the same day. Similar treatment repeated at 4 week interval after proper clinical evaluation of improvement of the lesion.

Exclusion criteria were the vitamin A derivatives use within 2 months of study initiation, any treatment procedure for Keloid including any type of laser modality within 1 year of study, pregnancy, use of immunosuppressive drugs, and any other diseases which can affect the wound-healing process. The protocol and informed consent were approved by the Department of Dermatology \& Venereology, Combined Military Hospital, Dhaka Cantonment, Dhaka.

\section{Treatment protocol:}

Pulsed dye laser

The laser procedure was performed in an outpatient setting because general anesthesia istypically unnecessary. Topical or intralesional anesthesia sometimes given in patients undergoing treatment to sensitive body areas (e.g., lips, breasts, perineum, 
fingers) as well as when treating younger patients. Topical lidocaine cream (e.g., EMLA, ElaMax) applied for 30 to 60 minutes to achieve adequate anesthesia. Immediately before laser treatment, anesthetic cream and make-up removed completely. Operative personnel, patients, and accompanying persons wear eye protection (e.g. goggles) appropriate to the 595$\mathrm{nm}$ wavelength. Flammable preparatory substances (e.g. alcohol, acetone) must not be applied on cutaneous surfaces to be treated because of their incendiary potential. $8,10,11$

\section{Laser parameters}

Appropriate energy fluencesfor keloids range from 6.0 to $7.5 \mathrm{j} / \mathrm{cm} 2$ with the-use of a 5 - or 7 $\mathrm{mm}$ spot size, and 4.5 to $5.5 \mathrm{j} / \mathrm{cm} 2$ with the use of a $10-\mathrm{mm}$ spot. Single laser pulses are delivered in an adjacent, nonoverlapping manner to cover the entire keloid.

\section{Postoperative management}

Purpura-associated color changes and swelling are seen at their most intense within the first 24 to 48 hours, and resolve over 7 to 10 days.Vesiculation, crusting, or bleeding are indicative of excessive fluences or overlapping of laser pulses which should be avoided in subsequent treatments. The severity of these adverse effects can be limited by the application of ice packs during the first few hours after surgery. The postoperative wound care regimen involves daily gentle cleansing with mild soap and water, followed by application of a healing or antibiotic ointment and a nonstick bandage. The treatment site is evaluated 3 to 4 weeks later. If the previous treatment yielded noticeable keloid improvement, the same fluence should be used again. In instances in which only minimal or no improvement is observed, the energy should be increased by $0.5 \pi \mathrm{cm} 2$

\section{Inj. Triamcinolone acetonide}

Intralesional triamcinolone is injected directly into the skin lesion using a fine needle after cleaning the site of injection with alcohol or antiseptic solution. The injection is intradermal, not subcutaneous, to avoid causing a dent in the skin. The initial dose per injection site is $40 \mathrm{mg} / \mathrm{ml}$ (full strength) or 0.1 mLper square centimeter of involved skin. ${ }^{8}$

\section{Assessment}

All patients tolerated the laser and conventional treatment well, and all keloids showed clinical improvement after $2^{\text {nd }}$ or $3^{\text {rd }}$ sessions at 4-week intervals. No complication such as permanent pigmentary change,ulceration or infection was observed in treatment course. High resolution digital photographs were taken using identical camera settings at the baseline and after completion of treatment. Assessment was done by using the Vancouver scar scale (VSS). VSS is composed of four components; vascularity, pigmentation, pliability and height. Each variable has four to six possible scores (table1). ${ }^{9}$ A total score ranges from 0 to 14 , whereby a score of 0 reflects normal skin.A caliper was used to determine the keloid height. For assessment of pigmentation and vascularity, we used a transparent tool for blanching the scar. Other values and complications, such as textural or discoloration (hypo- or hyperpigmentation) were assessed by a simple questionnaire. Overall evaluation has been done using the 'quartile grading scale' shown below with table -1 . Many patients appreciate the cessation of keloid-related symptoms such as pruritus and burning.

\section{Adverse effects}

The most common adverse effect of 595-nm pulsed dye laser treatment is transient hyperpigmentation, which occurs most often in darker pigmented individuals and in patients who did not follow strict precautions against sun exposure. Further laser treatment sessions should be delayed until all dyspigmentation has completely resolved. Prolonged erythema, vesiculation, and or pruritus of the treatment area should raise concerns of contact dermatitis, usually to a topical antibiotic. Other possible adverse reactions, including blister formation followed by secondary infection or keloidworsening, can be prevented by the use of appropriate laser parameters and avoidance of pulse overlap. ${ }^{7,8}$

\section{Data collection and analysis}

Clinical, laboratory and follow up findings for each individual patient were collected in a predesigned data collection sheet. Collected 
data were compiled and entered into computer based software, Statistical Package for Social Science (SPSS) for windows version 16.0. The categorical data was expressed as frequency, proportion and percentage. $\mathrm{P}$ value $<0.05$ was considered as minimum level of significance.

\section{Results:}

The majority of patients with keloid experienced up to $94 \%$ clinical improvementafter $2^{\text {nd }}$ or $3^{\text {rd }}$ session of treatment. Total 90 keloids were treated in 50 selected patients (18 female and 32 male). Mean age was 32.5 years ranging from 15 to 50 years. $78 \%$ (39 of 50 ) of the participants were male. The lesions were mainly located on sternum, shoulder, back and few on both extremities. Duration of their lesions ranged from 1 year to 10 years. At the end of $2^{\text {nd }}$ session of treatment there was significant improvement of lesions.

\section{Table-I}

Demographics of 50 patients with keloid

\begin{tabular}{lc}
\hline Male & 32 \\
\hline Female & 18 \\
Age range (years) & $15-50$ \\
Mean age & 32.5 years \\
Duration of lesion & 1year to 10 years \\
\hline
\end{tabular}

\section{Table-II}

Showing the magnitude of improvement in term of vascularity, height, pliability and hyperpigmentation before and after treatment

\begin{tabular}{lcc}
\hline & $\begin{array}{c}\text { Before } \\
\text { treatment }\end{array}$ & $\begin{array}{c}\text { After } \\
\text { treatment }\end{array}$ \\
\hline Vascularity & 2.7 & 0.61 \\
Pliability & 3.9 & 1.1 \\
Hyperpigmentation & 2.4 & 0.92 \\
Height & 2.87 & 0.78 \\
\hline
\end{tabular}

The mean vascularity of keloid before treatment was 2.7 and after this combined clinical trial mean score become 0.61. Pliability before treatment was 3.9 and after treatment 1.1, Hyperpigmentation before treatment was 2.4 and after treatment 0.92, height beforetreatment was 2.87 and after treatment 0.78 . The mean VSS score (0-14) of keloid decreased from 12.17 to a post treatment value of 3.41. Total 45.5 (91\%) patient showed excellent improvement, scored 4 of Quartile grading scale. $4.5 \%$ patient scored 2 to 3 of Quartile grading scale with a moderate to good improvement.

\section{Table-III}

Percentage of improvement of lesions after a 4 week combined treatment by using quartile grading scale

\begin{tabular}{ccc}
\hline $\begin{array}{c}\text { Quartile grading } \\
\text { scale }\end{array}$ & Improvement & Percentage \\
\hline 0 & No improvement & 0 \\
1 & Mild & 0 \\
2 & Moderate & 02 \\
3 & Good & 07 \\
4 & Excellent & 91 \\
\hline
\end{tabular}

\section{Discussion:}

Keloid can be disabling for patients because of ugly appearance and creation of social stress. The pulsed dye laser is recently recognized as an excellent first-line treatment option for treatment of keloid, although 5 years ago this treatment option might have been considered a viable choice only after all other methods failed. Many reports are present about effectiveness of PDL but good results in special race patients do not guarantee the same in other races. Some scientific studies on pulsed dye laser in the treatment of keloid and hypertrophic scar resulted in equivocal conclusion in Asians, Hispanic and Orientals. On this background PDL followed by I/L TAC proved most beneficial with an excellent outcome. Our study indicated that 3 or 4 session of treatment with PDL followed by intralesional TCA resulted in rapid decrease in VSS score in vascularity (from 2.7 to 0.61 ), in pliability (from 3.9 to 1.1 ), in hyperpigmentation (from 2.4 to 0.92), in height (from 2.87 to 0.78$)$. And finally the VSS score (0-14) of keloid decreased from 12.17 to a post treatment value of 3.41 which is an excellent achievement. 
Alster and Handrick found in their study which was done only with pulsed dye laser in 2007, the majority of patients with keloid experiencedup to $80 \%$ clinical improvement after 2 pulsed dye laser treatments. ${ }^{1}$

Omranifard and Rastishowed in their study where they compare the effects of conventional method, pulse dye laser and erbium laser for the treatment of hypertrophic scars and keloid in Iranian patients. Although the mean Vancouver scar scale (VSS) score significantly decreased in all three groups after treatment, the decrease in mean VSS score in PDL group was significantly lower than other groups. ${ }^{5}$

Alster and Williams performed a controlled study that examined 16 patients with sternotomykeloid and scar treated with pulsed dye laser. They detected significant degrees of improvement in terms of color and skin thickness when compared with the baseline and controls.

Morefibrotic or proliferative hypertrophic keloids typically require additional treatment sessions in order to obtain the desired degree of improvement. Only treatment with $40 \mathrm{mg}$ per $\mathrm{mL}$ TCA injected intralesionally, will eventually flatten 50 to 100 percent of keloids (depending on thickness and size of the keloid) with a 9 to 50 percent recurrence rate.

\section{Conclusion:}

Pretreatment with PDL facilitated steroid injection by making the keloid edematous and therefore softer. In addition, we speculate that the benefits of this combined modalities are summative, not just adjunctive. This study demonstrates that this modality is highly effective for the treatment of keloid when other treatments have been unsuccessful.

\section{References:}

1. Alster, TS, Handrick C. Laser treatment of hypertrophic scars, keloids, and striae. Semin Cutan Med Surg 2000; 19(4): 287-92.

2. Meenakshi J, Jayaraman V, Ramakrishnan KM, Babu M. Keloids and hypertrophic scars: a review. Indian J Plast Surg 2005; 38: 175-9.

3. Alster TS, Tinzi EL. Hypertrophic scars and keloids: etiology and management: Am J Clin Dermatol 2003; 4(4): 235-43.

4. Juckett G, Hartman-Adams H. Management of keloids and hypertrophic scars. Am Fam Physician 2009; 80(3): 253-60.

5. Omranifard M, Rasti M. Comparing the effects of conventional method, pulse dye laser and erbium laser for the treatment of hypertrophic scars in Iranian patients. J Res Med Sci 2007; 12: 277-81.

6. James WD, TG, Elston DM. Andrew's Disease of the skin-clinical dermatology. $10^{\text {th }}$ ed. USA: Saunders Elsevier; .

7. Asilian A, Darougheh A, Shariati F. New combination of triamcinolone, 5-Fluorouracil, and pulsed-dye laser for treatment of keloid and hypertrophic scars. Dermatol Surg 2006; 32(7): 907-15.

8. Kono T, Erçöçen AR, Nakazawa H, Honda T. The flashlamp-pumped pulsed dye laser (585 nm) treatment of hypertrophic scars in Asians. Ann Plast Surg 2003; 51(4): 366-71.

9. Draaijers LJ, Tempelman FRH, et al. The Patient and observer scar assessment scale: a reliable and feasible tool for scar evaluation. J Am Soc Plast Surg 2003; 113(7): .

10. Kono T, Ercocen AR, Nakazawa H, Honda T, Hayashi N, Nozaki M. The flashlamp-pumped pulsed dye laser (585 $\mathrm{nm}$ ) treatment of hypertrophic scars in Asians. Ann Plast Surg 2003; 51: 366-71.

11. Connell PG, Harland CC. Treatment of keloid scars with pulsed dye laser and intralesional steroid. J Cutan Laser Ther 2000; 2(3): 147-50. 